\title{
Cosmic rays IX
}

\section{Interactions and transport of cosmic rays in the Galaxy}

\author{
P. L. Biermann ${ }^{1,2}$, N. Langer ${ }^{3}$, Eun-Suk Seo ${ }^{4}$, and T. Stanev ${ }^{5}$ \\ 1 Max-Planck Institut für Radioastronomie, Auf dem Hügel 69, 53121 Bonn, Germany \\ 2 Department of Physics and Astronomy, University of Bonn, Bonn, Germany \\ 3 Dept. of Astronomy, Univ. of Utrecht, Utrecht, The Netherlands \\ 4 Institute for Physical Science and Technology, Univ. of Maryland, College Park, MD 20742, USA \\ 5 Bartol Research Institute, Physics Department, University of Delaware, Newark, DE 19716, USA \\ Received 3 August 2000 / Accepted 5 January 2001
}

\begin{abstract}
We propose that cosmic rays interact mostly near their sources of origin. To be specific, we differentiate the various supernovae by their mass of the progenitor star along the zero age main sequence. Stars between about 8 and 15 solar masses explode into the interstellar medium, and accelerate cosmic rays, as discussed by many for some time. From about 15 to 25 solar masses stars explode into their own stellar wind; this wind has built up a thin shell of both wind material and interstellar medium material in the red and blue giant phases preceding the supernova event. The shock accelerating cosmic ray particles races through that wind, gets loaded up with energetic particles, interacts while it goes, and finally smashes into the shell. While the shock goes out, it snowplows the entire wind into the pre-existing shell to form a composite shell. We propose that for the mass range 15 to 25 solar masses this composite shell is immediately broken up so that the time scale for interaction is caused by the breakup and so is convective. We note that the wind material for this range of zero age masses is a approximately half helium, and half hydrogen. The interactions in the composite wind-shell and the immediate environment produce positrons, gamma emission, but only few secondary nuclei, because for this mass range the enrichment in heavier elements is still minor. The energy spectrum of the gamma emission and the positrons produced corresponds then to the source spectrum. In contrast, from about 25 solar masses and up the wind is strongly enriched in heavy elements, and the wind shell is massive, comprising most of the initial zero age star's mass, as well as a good part of the local interstellar medium. We propose that for the interaction of the cosmic ray particles carried out by the shock in the snow-plow through the wind to the shell the interaction is diffusive, and calculate the diffusion coefficient. This leads to a leakage time energy dependence of $E^{-5 / 9}$ in the relativistic limit. This then gives an energy dependence of secondary nuclei, that matches the observations. There is a second component of positrons, and also gamma emission, but then at moderate energies all with the steeper energy dependence; spatial and velocity constraints give both a lower as well as an upper rigidity limit to the diffusion approximation. One important element in such a picture is the steady mixing of newly enriched material throughout the star before the explosion, induced by Voigt-Eddington circulation caused by rotation. The mixed material is then ejected through the wind, which at the end provides the source material for cosmic ray injection. This means that by the time the nuclei are subject to acceleration, they should have decayed already to final states, an effect which may be measureable in cosmic ray isotope ratios. Therefore, considering the history of the travel of cosmic rays through the normal interstellar medium, we can readily explain the ratio of secondaries to primaries, and at the same time use a spectrum of turbulence in the interstellar medium, a Kolmogorov spectrum, which is consistent with all other observational evidence. The escape time from the Galaxy is then proportional to $E^{-1 / 3}$ in the relativistic range of particle energies. Translating this result into the language common in the literature, this means that interaction path as measured in $\mathrm{gm} / \mathrm{cm}^{2}$ and escape time can not be used synonymously.
\end{abstract}

Key words. cosmic rays - cosmic ray transport - spallation - gamma spectrum - Galaxy

\section{Introduction}

Cosmic Rays are energetic particles, which come to us from outer space, and are measured either through satellites, balloons, or Earth based experiments. These

Send offprint requests to: P. L. Biermann, e-mail: plbiermann@mpifr-bonn.mpg.de energetic particles interact, and the result of these interactions are the spallation products as well as gamma rays. Gamma rays arise either from collisionally excited nuclei or nuclear fragments, or as a continuum from $\pi$-decay.

The origin of cosmic rays is still a question (Hess 1912; Kohlhörster 1913; Fermi 1949; Fermi 1954; Ginzburg \& Syrovatskij 1963; Ginzburg \& Syrovatskij 1969; 
Hayakawa 1969; Berezinsky et al. 1990) which is not finally settled. The debate has reached a consensus, that most are produced in the shockwaves of supernova explosions (Baade \& Zwicky 1934; Shklovskii 1953; Ginzburg 1953a, 1953b; Lagage \& Cesarsky 1983; Drury 1983; Blandford \& Eichler 1987; Berezhko \& Krymskii 1988; Jones \& Ellison 1991; Ginzburg 1993; Ginzburg 1996), be it into the interstellar medium, or into a stellar wind (Völk \& Biermann 1988; Silberberg et al. 1990; Biermann 1993a). Many of the relevant issues here have been dealt with in the review by Hillas (1984) and in the books by Hayakawa (1969), Berezinsky et al. (1990) and Gaisser (1990).

Here we are concerned with the interactions of cosmic rays (CRs) in the Galaxy, and so we will adopt the picture that indeed the cosmic ray particles originate in the shocks of supernova explosions, and thus limit ourselves to the corresponding energies. This paper is motivated by three arguments: 1) the wish to further develop the cosmic ray theory initiated some time ago (Biermann 1993a, 1993c), 2) by the apparent discrepancy between the observed gamma-spectrum of the Galaxy (Hunter et al. 1997) and the expected spectrum, and 3) by the apparent discrepancy between the turbulent spectrum required by standard cosmic ray transport theory (Garcia-Munoz et al. 1987), and the normal observations of the interstellar medium (ISM).

The structure of this paper is as follows: first we briefly summarize the proposal to account for the origin of cosmic rays; then we go through the arguments for interaction with the material close to the progenitor star; then we briefly address the issue where the interaction happens which produces the observed gamma ray emission from the Galaxy. Finally we draw some conclusion and make some specific predictions.

\section{A quantitative proposal for the origin of galactic cosmic rays}

Cosmic rays arrive at Earth with energies from several hundred $\mathrm{MeV} /$ particle to $310^{20} \mathrm{eV}$; their spectrum for protons is at $\mathrm{GeV}$ energies close to $E^{-2.75}$, and for $\mathrm{He}$ and higher elements close to $E^{-2.65}$ below a knee at $\approx 510^{15} \mathrm{eV}$, where the spectrum turns down to about $E^{-3.1}$, to flatten out again near $310^{18} \mathrm{eV}$, called the ankle (e.g. Lawrence et al. 1991; Nagano et al. 1992; Zatsepin 1995). The chemical composition is roughly similar to that of the interstellar medium, with reduced hydrogen and helium relative to silicon.

A proposal (see, e.g., Biermann 1993a, 1997a) is that three sites of origin account for the cosmic rays observed, i) supernova explosions into the interstellar medium, ISMSN, ii) supernova explosions into the stellar wind of the predecessor star, wind-SN, and iii) radio galaxy hot spots. Here, in this concept, the cosmic rays attributed to supernova-shocks in stellar winds, wind-SN, produce an important contribution at all energies up to $310^{9} \mathrm{GeV}$.
We note that the nucleosynthesis in Wolf-Rayet stars (e.g., Prantzos et al. 1987), and their contribution to cosmic rays has been discussed previously (Yanagita et al. 1990). It appears that they do make a substantial contribution, even as seen in the context of the standard leaky box model approach.

Particle energies go up to $100 \mathrm{Z}$ TeV for ISM-SN, and to $100 \mathrm{Z} \mathrm{PeV}$ with a bend at $600 \mathrm{Z} \mathrm{TeV}$ for wind-SN, the knee (Stanev et al. 1993), where $\mathrm{Z}$ is the charge of the nucleus considered. Radiogalaxy hot spots contribute up to about $100 \mathrm{EeV}$ at the source, with some sources up to $410^{21} \mathrm{eV}$ (Biermann 1997b). These numerical values are estimates with uncertainties of surely larger than a factor of 2 , since they derive from an estimated strength of the magnetic field, and estimated values of the effective shock velocity.

The spectra are predicted to be $E^{-2.75 \pm 0.04}$ for ISM$\mathrm{SN}$, and $E^{-2.67_{-0.04}^{+0.00}}$ for wind-SN below the knee, and $E^{-3.07_{-0.14}^{+0.00}}$ for wind-SM above the knee, and about $E^{-2.0}$ at injection for radiogalaxy hot spots.

These predictions can be compared at some detail with data, and we have given comparisons in previous work (e.g. Biermann 1993a; Biermann 1993b; Wiebel-Sooth et al. 1998; Biermann 1996; Biermann $1997 \mathrm{a}, 1997 \mathrm{~b})$. Newer data confirm some of the general trends near the knee (Glasmacher et al. 1999a; Glasmacher et al. 1999b).

\section{Spallation of cosmic ray nuclei}

Cosmic ray nuclei can be broken up in collisions with thermal matter; this process is called spallation. Obviously, there is a corresponding interaction between energetic protons, and thermal material comprising heavier nuclei such as carbon. In such collisions the remaining nuclei can also be excited, and then emit $\gamma$-ray lines.

Spallation is relevant in various sites (see, e.g., the recent work in this area, Garcia-Munoz et al. 1977; Engelmann et al. 1985; Garcia-Munoz et al. 1987; Engelmann et al. 1990; Shibata 1996 and the classical reviews by Reeves 1974, 1994). The site normally explored is interstellar clouds Spitzer (1968); cosmic rays travel through the interstellar medium, and interact. Good evidence for this interaction is the excellent correlation of the observed gamma ray continuum emission (arising from $\pi^{0}$ decay following p-p interaction) with the observed column density of interstellar material (Hunter et al. 1997).

\subsection{Source related spallation}

Massive stars eject all the material enriched over the big bang nucleosyntheis into the interstellar medium. Massive stars are also believed to be the sources of energetic particles; massive stars have powerful winds, which sweep up interstellar matter. Massive stars have so much mass loss, that they shed their outer layers, expose the processed material and thus late in life show a highly enriched wind. 
The wind, on the other hand, plows into the environmental gas, and sweeps it up; gas left over from the formation of the star may still be around as well, so as to increase the target for the expanding wind and form a shell. It is through this enriched wind and associated shell, that the supernova shock races, and causes particle acceleration and spallation by these particles directly. We note that the supernova shock compresses the wind itself into a shocked shell, which then smashes into the shell produced by the progenitor stellar wind. As the previous wind was also already enriched, spallation necessarily occurs in an enriched environment: such an enrichment is actually required by the light element data of old stars as shown by Ramaty et al. (1997), but would of course also be consistent with other scenarios than the one introduced here (the dust model mentioned below).

We note that the evolution of massive stars can now be modelled including the effects of rotation, the induced mixing and the strong winds (Heger \& Langer 1998; Langer et al. 1999); the mixing of the chemical element composition throughout the star during the evolution fixes the abundances in the wind, the site of acceleration upon explosion as a supernova. For the proposal as to the origin of cosmic rays it is a necessary ingredient, that these stars have fairly substantial magnetic fields (Thum \& Morris 1999; Seemann \& Biermann 1997).

Stars along the main sequence need to be considered in four separate zero age mass ranges:

- Stars below about $8 M_{\odot}$ do not explode as supernovae. This is obviously most stars;

- Stars from about 8 to about $15 M_{\odot}$ explode as supernovae, but do not have a strong stellar wind, and so explode into the interstellar medium;

- Stars from about 15 to $25 M_{\odot}$ have a substantial wind (Biermann et al. 1995); the wind is enriched only in helium. The chemical composition of the wind at the time of explosion is approximately 0.5 in He and 0.5 in $\mathrm{H}$. The mass in the shell of wind-swept material is moderate;

- Stars from about $25 M_{\odot}$ have a strong wind; the wind is enriched in heavy elements and has little hydrogen left. The mass in the shell of wind-swept material is large.

The last three mass ranges correspond roughly in numbers to a ratio of $3: 1: 1$, using a Salpeter law for the initial mass function.

Therefore we can ask the following question: what happens when the supernova shock smashes into the winddriven shell? The supernova shock shell is loaded with cosmic ray particles. In the enriched shock shell and wind shell the energetic particles may reside for some time, and then leak out into the normal interstellar medium. What is the wavefield that governs this leakage in the limit that the leakage is diffusive?

\subsubsection{The mass range above 25 solar masses}

Therefore, we need to consider the diffusive loss from a shell, which is traversed by a shock filled with a cosmic ray population. So we first derive the wavefield in magnetic fields excited by the cosmic rays. Then, in a second step we consider the diffusive leakage from the shell under the influence of this wave field.

Turbulence is an ubiquitous phenomenon, and also is a key ingredient in the interstellar medium (see reviews by Rickett 1990; Goldstein et al. 1995). Key concepts to turbulence theory have been introduced by Prandtl (1925), Karman \& Howarth (1938), Kolmogorov (1941a, 1941b, 1941c), Obukhov (1941), Heisenberg (1948), Kraichnan (1965), and have been reviewed by Sagdeev (1979). One key argument which we wish to use, is the concept of the turbulent cascade. In a cascade the energy of the turbulence is injected into the gas at some large wavelength, and cascades down through wavenumber space, to the small wavelengths where the energy is dissipated; in many examples this leads in a three-dimensional isotropic model to the Kolmogorov cascade (Matthaeus \& Zhou 1989), which can be described in a local approximation by the following diffusion equation in wavenumber space (McIvor 1977; Achterberg 1979).

The basic concept here is as follows: cosmic rays excite turbulence at all wavelengths through resonance, with the wavenumber $k=2 \pi / r_{\mathrm{g}}$, where $r_{\mathrm{g}}$ is the Larmor radius of a cosmic ray particle in the magnetic field. The entire spectrum of cosmic rays excites turbulence. This excitation takes place in the region near to and upstream from the super-nova shock, where there is a spatial gradient in cosmic ray flux. Downstream from the shock, where there is no more spatial gradient in the cosmic ray flux, the turbulence spectrum is given, and stays constant. A competing process to excitation is cascading, and the following equations describe this competition, with the first one showing cascading by itself:

$$
\frac{\partial}{\partial t} \frac{I(k)}{4 \pi k^{2}}-\frac{1}{k^{2}} \frac{\partial}{\partial k}\left(\frac{k^{4}}{3 \tau_{k}} \frac{\partial}{\partial k}\left(\frac{I(k)}{4 \pi k^{2}}\right)\right)=A \delta\left(k-k_{\mathrm{o}}\right) .
$$

Here $I(k)$ is the energy density of the turbulence per wavenumber $k$, and per volume element, and $\tau_{k}$ is the time scale of diffusion, which can be written as

$$
\tau_{k}=\frac{1}{k\left(\gamma_{\mathrm{eff}} I(k) k / \rho\right)^{1 / 2}}
$$

Here $\rho$ is the matter density, and $\gamma_{\text {eff }}$ is an effective adiabatic constant for the turbulent energy. The turbulence has a source-term, here limited to a single wavenumber $k_{o}$. Below we will consider an entire range of excitation wave numbers. The turbulence diffusion equation basically says that the turbulence moves through wavenumber space with no additional source or sink, as a constant 
energy current in wavenumber phase space (Kolmogorov 1941a; Kolmogorov 1941b; Kolmogorov 1941c). The solutions to this diffusion equation can be written as $I(k) \sim$ $k^{2}$ for $k \leq k_{\mathrm{o}}$ and $I(k) \sim k^{-5 / 3}$ for $k \geq k_{\mathrm{o}}$. This latter behaviour is commonly referred to as the Kolmogorov cascade, and is found ubiquitously in nature.

The basic equation for the excitation of wavefields can be written as follows (Bell 1978a; Drury 1983):

$$
\frac{\partial}{\partial t} \frac{I(k)}{4 \pi k^{2}}=\left(\sigma_{\text {excit }}-\Gamma_{\text {damp }}\right) \frac{I(k)}{4 \pi k^{2}}
$$

with $\sigma_{\text {excit }}$ the excitation term and $\Gamma_{\text {damp }}$ the damping term. Here we will concentrate on the excitation of waves, and ignore any damping. The excitation term can be written as

$\sigma_{\text {excit }}=\frac{4 \pi}{3} \frac{v_{\mathrm{A}}}{I(k) k} p^{4} v \frac{\partial f}{\partial x}$

where $v$ is the particle velocity, $f$ the particle distribution function in phase space (assumed to be isotropic), and $x$ the local coordinate perpendicular to the shock plane. We need to emphasize that only the difference of the particle spectrum across a shock wave or any other spatial gradient can excite waves; therefore, the first shock wave loaded with cosmic ray particles sweeping across the molecular gas shell around exploding stars and their winds is the excitation mechanism, which we consider. Now the particle spectrum in energy space (in the relativistic domain of particle speeds) is $N(E)=E^{-7 / 3}$ from the arguments of shocks in winds (Biermann 1993a; Biermann \& Cassinelli 1993). Of course, this step in the argument depends on the proposal for the origin of cosmic rays summarized earlier to be correct. This earlier argument entails that the length scale inherent in the upstream gradient of cosmic rays is independent of energy, different in this subtle point from Bell (1978a). We defer the combined solution of the cascade equations and the cosmic ray kinetic equations to later work, since for such an endeavour the highly turbulent shock region needs to be modelled in much more detail than possible at this stage.

We therefore need to determine which wave spectrum can balance the cascading with the excitation from the freshly injected cosmic rays. Equating the two expressions above for the cascading (Eq. (1)) and the excitation (Eq. (3)) in their $k$-dependence using an arbitrary powerlaw for $I(k) \sim k^{-\beta}$ then yields the condition $-\frac{1}{2}-\frac{3}{2} \beta=\frac{7}{3}-5$ which means $\beta=\frac{13}{9}$. We omit here for lack of space a thorough discussion of the errors in this line of argument, as in e.g. (Biermann \& Cassinelli 1993; Biermann 1997a; Wiebel-Sooth et al. 1998). From (Drury 1983) we obtain the diffusion coefficient based on the wavefield, and then the diffusive time scale of loss from the diffusion coefficient in turn.
To emphasize the argument here: the spatial gradient near to and upstream of the supernova shock excites turbulence at all wavelengths through resonance between waves and the Larmor motion of cosmic ray particles; the turbulent spectrum is established by competition between excitation and cascading. In the downstream region then the turbulent spectrum is given, stays constant, and determines the post-shock leakage of cosmic rays from the region.

The diffusion coefficient in the quasilinear appproximation is given by

$\kappa=\frac{1}{3}(\beta c) r_{\mathrm{g}} \frac{B^{2} / 8 \pi}{I(k) k}$.

This entails an energy dependence of the leakage time from the zone of interaction

$\tau_{L} \sim \beta^{-1}(p c)^{-5 / 9}$

for $p_{\min , 1} / m_{\mathrm{e}} c<p<p_{\max } / m_{\mathrm{e}} c$ where $p c \sim r_{\mathrm{g}} \sim 1 / k$ is the momentum of the particle multiplied with the speed of light, so the energy in the relativistic limit. However, in order to allow a discussion also below relativistic energies, we use here the trans-relativistic description, which requires the momentum. This then means that the local spectrum of primary cosmic rays is steeper by this much, and so all secondaries are produced with a spectrum which is steeper by this much from the start.

$p_{\min , 1}$ is a critical momentum, below which the convective break up induced by the shock takes over, and the time scale becomes independent of particle momentum. There is also an upper limit for the validity of the diffusion approximation, $p_{\max }$, where the diffusion time becomes equal to the travel time of particles at the speed of light through the region of interaction. Beyond that limit again, the interaction time becomes a constant with energy. So, the diffusion approximation has both lower and upper limits for the transrelativistic momentum. Outside these limits the interaction time is constant with particle energy. The momentum (or "rigidity") range between these two limits should therefore be of order 10 to 30 . Obviously, at $p_{\min , 1}$ and at $p_{\max }$ the time scale has to be continuous.

The grammage traversed then is given by the product of this time scale multiplied with the average density in the interaction region, and also multiplied with the particle velocity:

$\lambda_{\text {int }} \sim \beta \tau_{\mathrm{L}}$

cancelling one $\beta$-dependence. Thus, in the (zero age) mass range of stars considered here, above 25 solar masses, the 
grammage traversed as a function of energy is first proportional to $\beta$, then $p^{-5 / 9}$, and finally merges into a constant.

The grammage can be estimated from the observed boron/carbon $(\mathrm{B} / \mathrm{C})$ ratio in the $\mathrm{CR}$-data using the point where the $E^{-0.6}$ breaks down, and the $\mathrm{B} / \mathrm{C}$ ratio becomes consistent with a constant for higher energies. Integrating through a stellar wind of a WR-star and through the winddriven shell to get the total interacting material one indeed obtains a grammage consistent with the requirements from the data, of order $10 \mathrm{gm} / \mathrm{cm}^{2}$, as required by the data.

Therefore, within the energy range of validity of the diffusion approximation, secondaries are produced with a spectrum of

$$
N_{\text {sec }}(p c) \sim(p c)^{-26 / 9}
$$

\subsubsection{The mass range between 15 and 25 solar masses}

For the stars between about 15 and 25 solar masses, we have a thin non-diffusive shell, where the escape time is just proportional to the inverse particle velocity

$\tau_{\mathrm{L}} \sim(\beta)^{-1}, \quad$ for $\quad p>p_{\min , 2}$,

or again, if the general convective velocities are larger than the particle velocity, then the leakage time from the shell becomes independent of particle energy for $p<p_{\min , 2}$ (the index " 2 " is here to make obvious that this minimum momentum may be different in the two stellar mass ranges). Thus, in the (zero age) mass range of stars considered here, between 15 and 25 solar masses, the grammage traversed as a function of energy is first proportional to $\beta$, and then becomes a constant.

Considering again the $\mathrm{CR}$-data for the $\mathrm{C} / \mathrm{B}$ ratio as a function of energy, we note that the stars in this mass range do not contribute any significant new carbon from their wind, just the carbon already present in the original gas from which the star formed.

\subsubsection{Transport through the Galaxy}

After transport through the Galaxy and insignificant further spallation this means that the ratio of secondaries to primaries remains at

$$
N_{\text {sec }} / N_{\text {prim }} \sim(p c)^{-5 / 9}
$$

which is rather close to the observed dependence from the boron to carbon ratio of $E^{-0.6}$ (Engelmann et al. 1990).

Clearly, as expounded above, this treatment breaks down at two ends, both on the low energy side, and on the high energy side. On the high energy side, it is obvious that from some energy the diffusive approximation breaks down, and from the low energy end it is clear that convective losses and the full break up of the shell will be faster than diffusion below some energy.

There is necessarily also some interaction during the wandering through the interstellar medium (ISM): this interaction can be estimated by observing that the cosmic ray disk is at least $2 \mathrm{kpc}$ thick in full width, while the cold gas disk is about $100 \mathrm{pc}$ only. The average density in the cold gas disk is about 1 particle per cc, and so the average density as seen by the cosmic rays is about 0.05 per cc, and so of interest only at high energy. This interaction is governed by the Kolmogorov spectrum, and so those secondaries should be steeper than the primaries by $1 / 3$ in spectrum. The total grammage traversed through the ISM by particles near GeV can thus be estimated as near $2.5 \mathrm{gm} / \mathrm{cm}^{2}$.

The picture presented in the earlier sections of this paper, however, is in contrast to the usual finding that a stationary leaky box gives a ratio of secondary to primaries $\sim(p c)^{-1 / 3}$, if we use a Kolmogorov spectrum for turbulence; this latter result is obtained, when the interaction happens evenly throughout the residence period in the Galaxy.

This is also in contrast to the concept of clouds capturing and then releasing cosmic ray particles derived in (Biermann 1996).

Therefore, the effective interaction path is the sum of the composite shell interaction and the ISM, in some cases for the stars above 25 solar masses, and in some cases for the stars between 15 and 25 solar masses, while for the stars between about 8 and 15 solar masses the interstellar medium dominates.

\subsubsection{Escape time versus interacting path}

The cosmic rays observed are the sum of the various CRpopulations arising from explosions of stars in the three mass ranges considered here separately.

Therefore, considering the history of the travel of cosmic rays through the normal interstellar medium (see also, e.g. Biermann 1996; Pohl et al. 1997), we can readily explain the ratio of secondaries to primaries, and at the same time use a spectrum of turbulence which is consistent with all other observational evidence. Translating this result into the language common in the literature, this means that interaction path as measured in $\mathrm{gm} / \mathrm{cm}^{2}$ and escape time can not be used synonymously. The escape time is given by $\tau_{\mathrm{L} \text {,gal, }}$ and is proportional to $E^{-1 / 3}$ in the relativistic range of particle energies. The interaction path as a means to describe interaction is here $\sim(p c)^{-5 / 9} \mathrm{gm} / \mathrm{cm}^{2}$. The real time corresponding to the interaction phase is surely very short compared to the leakage time scale. 


\section{Predictions}

\subsection{Injection of cosmic rays}

Injection of cosmic rays from the wind-supernovae takes place in the stellar wind, which has a typical temperature due to cooling of around $20000 \mathrm{~K}$. Therefore many elements with low first ionization potential will be doubly ionized, and so should be preferred in injection. Therefore we expect that those elements will be enhanced in the cosmic ray abundances.

This is in contrast to another well developed model of injecting cosmic rays from spallation of energetic dust particles (Ellison et al. 1997; Meyer et al. 1997; Ramaty et al. 1997). What are the differences observationally between these two models?

- The dust model uses a spectrum of interstellar turbulence relevant for the transport of cosmic ray particles, which is inconsistent with a Kolmogorov spectrum, a model more consistent with a Kraichnan spectrum $\left(I(k) \sim k^{-3 / 2}\right)$, which is considered to be supported by the energy dependence of the $\mathrm{B} / \mathrm{C}$ ratio as a function of energy. Therefore, the model uses an injection spectrum of $E^{-2.1 \ldots 2.2}$. The Kraichnan spectrum is valid for a medium, which is made stiff in one dimension by a strong magnetic field. In the context of the standard leaky box model this allows a straightforward interpretation of the B/C-ratio, but it is only marginally consistent with the cosmic ray electron spectrum, which suggests an injection spectrum near $E^{-2.3}$;

- The dust model implies a gamma ray spectrum arsing from $\pi^{0}$-decay as a result of pp-collisions corresponding to the average spectrum of CRs of about $E^{-2.7}$, and so is inconsistent with the EGRET data. This could be remedied by argueing that the EGRET gamma spectrum is due to inverse Compton exclusively; such an argument has some strength because the EGRET emission extends to fairly high latitudes in the Galaxy. The gamma spectrum based on the model here will be discussed separately (Rhode et al. in preparation);

- The dust model clearly predicts different ratios of radioactive isotopes than the model presented here, since the interaction is en route between source and observer, whereas here the interaction is all near the source. This will be discussed elsewhere. This also applies to the positron/electron and anti-proton/proton ratios.

\subsection{Chemical abundances}

The abundances of cosmic rays is predicted to correspond to the abundances in the main acceleration sites, in the stellar winds for all elements from helium. Therefore the prediction has been that all these primary elements should have the spectrum $E^{-2.67_{-0.04}^{+0.00}}$ below the knee, and correspondingly $E^{-3.07_{-0.14}^{+0.00}}$ above the knee. The knee itself is at a given rigidity and so is predicted to show a gradual change from the lower abundances to enriched abundances
(Stanev et al. 1993; Glasmacher et al. 1999a, 1999b). We furthermore predict that a major component of the cosmic ray abundances should be traceable to the abundances in the stellar winds, folded appropiately with the number of stars along the main sequence. This will be elaborated on in a separate paper (CRVIII).

\subsection{Isotopic ratios}

Nuclear reactions produce various chemical elements and their isotopes, which then get transported through the star, escpecially in stars at high rotation. This transport is due to the Voigt-Eddington circulations induced by rotation. The mixing then brings the various isotopes and chemical elements to the base of the wind zone, and then they get transported out with the wind. Therefore, any decay of these nuclei has some time to have happened before the star explodes as a supernova, and the shock races through the wind, accelerating particles to become cosmic rays. The observed isotope ratios (e.g. Connell \& Simpson 1997) can be tested against such a prediction.

\subsection{The gamma ray spectrum of the Galaxy}

It has been argued for decades that the Galaxy should emit gamma rays from the decay of $\pi^{0}$ particles resulting from collisions of cosmic ray protons, and interstellar medium protons, with a probably small contribution from nuclear collisions of higher element nuclei such as helium (much of the early work was done by Stecker 1971). The data originally collected appeared to confirm this hypothesis very nicely. One could even fit in detail the bumps and wiggles of the column density of the interstellar medium with the gamma ray emission, confirming rather well and quantitatively the expectations of the model. There has been a worry for a long time, that in such a fit, the radial variation of the cosmic rays deduced appeared to be small, while we believe to know from other galaxies, that at least the electron component has a clear radial drop off, and so one might think the proton component ought to show such a gradient as well.

Recently the gamma ray spectrum of the Galaxy has been measured much better with the EGRET satellite (Hunter et al. 1997). It turned out, that once again, the spatial variation of the gamma ray emission can be fit rather well, while the spectrum can not be fitted; the authors in that paper used the approach that the local cosmic ray intensity is correlated with the local gas density, while as the cosmic ray spectrum they used the spectrum measured at Earth (demodulated from Solar wind effects). The observed gamma ray spectrum is much too flat to match the expectation based on the average oberseved cosmic ray spectrum - as deduced from either direct measurements or from radio data, once again connecting electrons and protons in this argument; below a few $\mathrm{GeV}$ this may be compensated for by invoking various models for inverse Compton emission and Bremsstrahlung, but at 
energies beyond $10 \mathrm{GeV}$ the discrepancy becomes quite pronounced. This has caused quite a stir, but sofar no obvious solution appears to be convincing (Mori 1997; Pohl et al. 1997).

The gamma ray emission can be fitted very well with a proton spectrum which is quite a bit flatter than the observed proton spectrum (Gaisser et al. 1998; Protheroe \& Stanev 1997), perhaps suggesting that we see the sources of cosmic rays. The picture suggested here would lead to the following.

As one goes up the main sequence from about $15 M_{\odot}$, the mass in the shell produced by the stellar wind increases slowly, to become quite substantial for very high mass stars at the end of their lifetimes, just before the stars explode. Therefore, one might expect that the leakage of cosmic ray particles may not be diffusive for lower stellar masses. However, there are many more lower mass stars, and so the $\pi^{0}$-producing collisions are dominated by the interaction in the shells of the lower mass stars, where the leakage is probably convective, and not diffusive. Then the interaction is with the primary spectrum, and so the gamma ray spectrum is predicted to match a proton (and helium) energy spectrum of $E^{-7 / 3}$. This is consistent with the gamma-ray spectral fit, which shows a minimum $\chi^{2}$-fit in a spectral range between 2.3 and 2.4 (Rhode et al., in preparation).

\subsection{Positrons}

In the wind shells around stars of the mass range above 15 solar masses gamma rays are produced from ppinteractions; in the same channel we produce positrons. Therefore we predict the positrons to to arise from two contributions: for the mass range 15 to 25 solar masses positrons are produced with a spectrum identical to the source spectrum, and for the mass range above about 25 solar masses positrons are produced with a spectrum which is $E^{-5 / 9}$ steeper. The sum of these two contributions needs to be balanced against the three contributions for electrons: a) electrons arise directly from those supernovae that explode into the interstellar medium, with a predicted spectrum of $E^{-2.74 \pm 0.04}$. As noted earlier (Biermann \& Strom 1993) the electrons from supernovae that explode into the interstellar medium, suffer from adiabatic losses, and so their contribution does not fully reflect the abundance of the progenitor stars. b) Electrons are both accelerated and also produced in pp-collisions as secondaries with a spectrum of $E^{-2.67_{-0.04}^{+0.00}}$ in the convective interaction mode in the wind shells around stars of zero age mass 15 to 25 solar masses. c) The stars with higher mass have a diffusive interaction for energetic particles in their wind shell and so make primary electrons again with $E^{-2.67_{-0.04}^{+0.00}}$, and secondary electrons and positrons with $E^{-26 / 9}$, where we omit the uncertainties. This will be discussed separately (Paper CR VI).

\subsection{Anti-protons}

Anti-protons have the again pp-collisions as sources, but of course get produced only at some higher energy. Both zero age mass ranges, 15-25 solar masses, and above 25 solar masses, again contribute just as for positrons, and with the corresponding spectra. This will be discussed separately (Sina et al., in preparation).

\subsection{Radioactive isotopes}

Radioactive nuclei that arise as a consequence of spallation decay. Since the production is predicted here to be happening in the thick wind shells around very massive progenitor stars, the decay has all subsequent time available from diffusive leakage from the Galaxy. For this diffusive leakage we adopted the description based on a Kolmogorov spectrum for the turbulence. The recent data have been discussed by Connell \& Simpson (1997), Simpson \& Connell (1998), Connell (1998), and Connell et al. (1998). In the model presented here the interaction is all the same, independent of isotope, and since we measure at rather low particle energy, almost certainly in the energy range, where the transport in the interaction shell is convective (and, presumably, also the interstellar transport), and therefore the ratios are not expected to depend on energy except through time-dilation at relativistic speeds. However, the initial ratio of the isotopes is fixed as they leave the source region, and the ratio changes as the particles are transported through the Galaxy, and therefore the grammage seen by the particles should all be identical, and their isotope ratio should just reflect the different times available. With the data as available, no differentiation to a standard leaky box model is possible. We will develop this approach separately.

\subsection{High energy gamma ray data}

One of the strongest tests for this model is the calculation of the expected gamma ray emission at high $\mathrm{TeV}$ and $\mathrm{PeV}$ photon energies, to be compared with CASA-MIA data and future MILAGRO data. While we have done some preliminary tests of this (to be published) that encourage us, we have not finished this test. For this test the bend, the knee, in the cosmic ray spectrum has to be taken into account, since in this model it is there already in the source.

\section{Outlook}

Here we have described an approach for cosmic ray interaction, which places most of the interactions near the site of origin, and have summarized some the various predictions which follow. We will go through these predictions quantitatively and check them off in detail against data in further papers. The key is the suggestion that the transport of energetic particles is diffusive in the thick composite shells around the wind of progenitor stars of 
a mass larger than about 25 solar masses. Those stars also are predicted to contribute cosmic rays all the way to $310^{18} \mathrm{eV}$; in this concept the bend in the cosmic ray spectrum known as the "knee" is caused by a decrease in the acceleration efficiency at a specific rigidity (i.e., at a specific Larmor radius), and so the bend is there already in the source (Biermann 1993a; Biermann \& Cassinelli 1993; Biermann 1997a). Those stars produce most of the heavy elements in this concept, and also most of the secondary nuclei. The contribution to gamma ray emission, positrons, anti-protons, and neutrons is all due to a combination of the interactions in the shells around stars in the mass range 15 to 25 solar masses, where the interaction is predicted to be convective, and the stars of higher mass, where the interaction is predicted to be diffusive.

Acknowledgements. The work has benefitted from interactions and collaborations with Venya Berezinsky, Elly Berkhuijsen, Jim Connell, Alina and Fanel Donea, Torsten Enßlin, Glennys Farrar, Tom Gaisser, Stan Hunter, Bob Kinzer, Hartmut Machner, Norbert Magnussen, Karl Mannheim, Sera Markoff, Bill Matthaeus, Jim Matthews, Hinrich Meyer, Dietrich Müller, Biman Nath, Ray Protheroe, Giovanna Pugliese, Jörg Rachen, Reuven Ramaty, Wolfgang Rhode, John Simpson, Ramin Sina, Friedel Thielemann, Clemens Thum, Alan Watson, Barbara Wiebel-Sooth, and Christian Zier. An early version of some of these ideas was presented at a nuclear physics conference (Hirschegg 1998). Cosmic Ray work with E.-S.S. is supported by a NASA-grant, high energy work with P.L.B has been supported by a grant from DESY, and common work by P.L.B. and T.St. is supported by a NATO grant.

\section{References}

Achterberg, A. 1979, A\&A, 76, 276

Baade, W., \& Zwicky, F. 1934, Proc. Nat. Acad. Science, 20(5), 259

Bell, A. R. 1978a, MNRAS, 182, 147

Berezhko, E. G., \& Krymskii, G. F. 1988, Sov. Phys. Usp., 31, 27

Berezinsky, V. S., et al. 1990, Astrophysics of Cosmic Rays (North-Holland, Amsterdam)

Biermann, P. L. 1993a, A\&A, 271, 649, Paper CR I

Biermann, P. L., \& Cassinelli, J. P. 1993, A\&A, 277, 691, Paper CR II

Biermann, P. L., \& Strom, R. G. 1993, A\&A, 275, 659, Paper CR III

Biermann, P. L. 1993b, in Currents in Astrophysics and Cosmology, Conf. 1990, ed. G. G. Fazio, \& R. Silberberg (Cambridge Univ. Press, Cambridge, UK), 12

Biermann, P. L. 1993, in 23rd ICRC, Invited, Rapporteur and Highlight Papers, ed. D. A. Leahy, et al. (World Scientific, Singapore, 1993), 45

Biermann, P. L., Gaisser, T. K., \& Stanev, T. 1995, Phys. Rev., D 51,3450

Biermann, P. L. 1996, in Proc. Nuclear Physics meeting MESON96 (Cracow), ed. E. Grosse, et al., Jagiellonian University, Acta Physics Polonica, B27, 3399

Biermann, P. L. 1997a, in Cosmic Winds and the Heliosphere, ed. J. R. Jokipii, et al. (University of Arizona Press, Tucson), 887

Biermann, P. L. 1997b, J. Phys. G, 23, 1
Biermann, P. L. 1998, in Proc. Nuclear Astrophysics, Hirschegg, GSI, Darmstadt, 211

Blandford, R. D., \& Eichler, R. D. 1987, Phys. Rep., 154, 1

Connell, J. J., \& Simpson, J. A. 1997, ApJ, 475, L61

Connell, J. J. 1998, ApJ, 501, L59

Connell, J. J., DuVernois, M. A., \& Simpson, J. A. 1998, ApJ, 509, L97

Drury, L. O'C. 1983, Rep. Prog. Phys., 46, 973

Ellison, D. C., Drury, L. O'C., \& Meyer, J.-P. 1997, ApJ, 487, 197

Engelmann, J. J., et al. 1985, A\&A, 148, 12

Engelmann, J. J., et al. 1990, A\&A, 233, 96

Fermi, E. 1949, Phys. Rev. 2nd Ser., 75(8), 1169

Fermi, E. 1954, ApJ, 119, 1

Gaisser, T. K. 1990, Cosmic Rays and Particle Physics (Cambridge Univ. Press)

Gaisser, T. K., Protheroe, R. J., \& Stanev, T. 1998, ApJ, 492, 219

Garcia-Munoz, M., Mason, G. M., \& Simpson, J. A. 1977, ApJ, 217,859

Garcia-Munoz, M., Simpson, J. A., Guzik, T. G., Wefel, J. P., \& Margolis, S. H. 1987, ApJS, 64, 269

Ginzburg, V. L. 1953a, Usp. Fiz. Nauk, 51, 343; see also 1956, Nuovo Cimento, 3, 38

Ginzburg, V. L. 1953b, Dokl. Akad. Nauk SSSR, 92, 1133 (NSF-Transl. 230)

Ginzburg, V. L., \& Syrovatskii, S. I., The origin of cosmic rays (Pergamon Press, Oxford 1964; Russian edition 1963)

Ginzburg, V. L., \& Syrovatskij, S. I. 1969, The Origin of Cosmic Rays (Gordon and Breach, New York)

Ginzburg, V. L. 1993, Phys. Usp., 36, 587

Ginzburg, V. L. 1996, Uspekhi Fizicheskikh Nauk, 166, 169

Glasmacher, M. A. K., et al. 1999a, Astropart. Phys., 10, 291

Glasmacher, M. A. K., et al. 1999b, Astropart. Phys., 12, 1

Goldstein, M. L., Roberts, D. A., \& Matthaeus, W. H. 1995, ARA\&A, 33, 283

Hayakawa, S. 1969, Cosmic Ray Physics (Wiley-Interscience, New York)

Heger, A., \& Langer, N. 1998, A\&A, 334, 210

Heisenberg, W. 1948, Z. Physik, 124, 628

Hess, V. F. 1912, Phys. Z., 13, 1084

Hillas, A. M. 1984, ARA\&A, 22, 425

Hunter, S. D., et al. 1997, ApJ, 481, 205

Jones, F. C., \& Ellison, D. C. 1991, Space Sci. Rev., 58, 259

Karman, Th. de, \& Howarth, L. 1938, Proc. of the Royal Soc. of London, 164, 192

Kohlhörster, W. 1913, Phys. Z., 14, 1153

Kolmogorov, A. N. 1941a, Dokl. Akad. Nauk SSSR, 30, 299

Kolmogorov, A. N. 1941b, Dokl. Akad. Nauk SSSR, 31, 538

Kolmogorov, A. N. 1941c, Dokl. Akad. Nauk SSSR, 32, 19

Kraichnan, R. H. 1965, Phys. Fl., 8, 1385

Lagage, P. O., \& Cesarsky, C. J. 1983, A\&A, 118, 223

Langer, N., et al. 1999, A\&A, 346, L37

Lawrence, M. A., et al. 1991, J. Phys. G, 17, 733

McIvor, I. 1977, MNRAS, 178, 85

Matthaeus, W. H., \& Zhou, Y. 1989, Phys. Fl., B1, 1929

Meyer, J.-P., Drury, L. O'C., \& Ellison, D. C. 1997, ApJ, 487, 182

Mori, M. 1997, ApJ, 478, 225

Nagano, M., et al. 1992, J. Phys. G, 18, 423

Obukhov, A. M. 1941, Dokl. Akad. Nauk SSSR, 32, 22

Pohl, M., et al. 1997, ApJ, 491, 159

Prandtl, L. 1925, ZAMM, 5, 136 
Prantzos, N., Arnould, M., \& Arcoragi, J.-P. 1987, ApJ, 315, 209

Protheroe, R. J., \& Stanev, T. 1997, at the ICRC meeting in Durban, South Africa, OG 3.4.3

Ramaty, R., Kozlovsky, B., Lingenfelter, R. E., \& Reeves, H. 1997, ApJ, 488, 730

Reeves, H. 1974, ARA\&A, 12, 437

Reeves, H. 1994, Rev. Mod. Phys., 66, 193

Rickett, B. J. 1990, ARA\&A, 28, 561

Sagdeev, R. Z. 1979, Rev. Mod. Phys., 51, 1

Seemann, H., \& Biermann, P. L. 1997, A\&A, 327, 273

Shibata, T. 1996, 24th Internat. Cosmic Ray Conf., rapporteur talk, Nuovo Cimento, 19C, 713

Shklovskii, I. S. 1953, Dokl. Akad. Nauk SSSR, 91(3), 475 (Lib. of Congress Transl. RT-1495)
Silberberg, R., et al. 1990, ApJ, 363, 265

Simpson, J. A., \& Connell, J. J. 1998, ApJ, 497, L85

Spitzer, L. Jr. 1968, Diffuse Matter in Space (Interscience Publishers, Wiley, New York)

Stanev, T., Biermann, P. L., \& Gaisser, T. K. 1993, A\&A, 274, 902, Paper CR IV

Stecker, F. W. 1971, Cosmic Gamma Rays, NASA SP-249

Thum, C., \& Morris, D. 1999, A\&A, 344, 923

Völk, H. J., \& Biermann, P. L. 1988, ApJ, 333, L65

Wiebel-Sooth, B., Biermann, P. L., \& Meyer, H. 1998, A\&A, 330, 389, Paper CR VII

Yanagita, S., Nomoto, K., \& Hayakawa, S. 1990, Proc. 21st ICRC, Adelaide, Australia, ed. R. J. Protheroe (Univ. of Adelaide, 1990), 4, 44

Zatsepin, V. I. 1995, J. Phys. G, 21, L31 